\title{
TriGen: A genetic algorithm to mine triclusters in temporal gene expression data
}

\author{
D. Gutiérrez-Avilés, C. Rubio-Escudero, F. Martínez-Álvarez, J.C. Riquelme
}

Keywords: Tricluster Genetic algorithms Microarray data Time series

\begin{abstract}
A B S T R A C T
Analyzing microarray data represents a computational challenge due to the characteristics of these data. Clustering techniques are widely applied to create groups of genes that exhibit a similar behavior under the conditions tested. Biclustering emerges as an improvement of classical clustering since it relaxes the constraints for grouping genes to be evaluated only under a subset of the conditions and not under all of them. However, this technique is not appropriate for the analysis of longitudinal experiments in which the genes are evaluated under certain conditions at several time points. We present the TriGen algorithm, a genetic algorithm that finds triclusters of gene expression that take into account the experimental conditions and the time points simultaneously. We have used TriGen to mine datasets related to synthetic data, yeast (Saccharomyces cerevisiae) cell cycle and human inflammation and host response to injury experiments. TriGen has proved to be capable of extracting groups of genes with similar patterns in subsets of conditions and times, and these groups have shown to be related in terms of their functional annotations extracted from the Gene Ontology.
\end{abstract}

\section{Introduction}

The use of high throughput processing techniques has revolutionized the technological research and has exponentially increased the amount of data available [11]. Particularly, microarrays revolutionized biological research by its ability to monitor changes in RNA concentration in thousands of genes simultaneously [7].

A common practice when analyzing gene expression data is to apply clustering techniques, creating groups of genes that exhibit similar expression patterns [31]. These clusters are interesting because it is considered that genes with similar behavior patterns can be involved in similar regulatory processes [34]. Although in theory there is a big step from correlation to functional similarity of genes, several articles indicate that this relation exists [10]. Traditional clustering algorithms work on the whole space of data dimensions examining each gene in the dataset under all conditions tested.

Applying clustering algorithms on gene expression data usually does not provide the best results. Much of the activity patterns of gene groups are only present under a particular set of experimental conditions. Actually, the available knowledge on cell processes suggests that, while a subset of genes is co-regulated and co-expressed under particular experimental conditions, under different conditions these genes can show independent behavior. Discovering this local behavior patterns can be the key to discover gene pathways, which could be hard to discover in other ways. For this reason, the paradigm of clustering techniques must change to methods that allow local pattern discovery in gene expression data [6].

Biclustering addresses this problem [16] by relaxing the conditions and by allowing assessment only under a subset of the conditions of the experiment, and it has proved to be successful in finding gene patterns [21]. However, clustering and biclustering are insufficient when analyzing data from microarray experiments where attention is drawn on how time affects gene's behavior [13]. There is a lot of interest in this type of longitudinal experiments because they allow an in-depth analysis of molecular processes in which the time evolution is important, for example, cell cycles, development at the molecular level or evolution of diseases [4]. Therefore, the use of specific tools for data analysis in which genes are evaluated under certain conditions considering the time factor becomes necessary. In this sense, triclustering appears as a valuable tool since it allows for the assessment of genes under a subset of the conditions of the experiment and under a subset of time points.

Biclustering is known to be a NP hard problem [35] and therefore many proposed successful biclustering algorithms are based on heuristics $[3,25]$. Since the computational complexity of 
triclustering is greater than the biclustering one, heuristic based algorithms are a good approximation for triclustering.

In this context, triclustering algorithms appear as a way to find genes of similar expression profiles along a segment of time series in a subset of conditions. We define a coherent tricluster as a set of genes which exhibits either similar numeric values for the times and conditions (coherent values [21]) or similar behaviors regardless of the exact numeric values: correlated positive and negative changes in the expression values (coherent behavior [21]). Both types of coherent clusters may contain information useful to identify useful phenotypes, potential genes related to these phenotypes and their regulation relations [36].

We present the TriGen (Triclustering-Genetic based) algorithm based on an evolutionary heuristic, genetic algorithms, which finds groups of pattern similarity for genes on a three dimensional space, thus taking into account the gene, conditions and time factor. Although many clustering and biclustering models define similarity based on distance functions [12,37], these functions are not always adequate to capture similarities among genes, since correlations may still exist among a set of genes which are expressed at different levels of magnitude. Therefore we propose two different evaluation functions: the first one finds triclusters of coherent values and is based on a three dimensions adaptation of the Mean Square Residue measure (MSR) which is a classic biclustering distance measure for gene expression analysis [9], the second one is a correlation measure that identifies triclusters of coherent behavior based on the least square approximation (LSL) which calculates the distances among the slopes of the least square lines from a tricluster.

We show that the results obtained from applying the TriGen algorithm to a synthetic dataset and two real ones: the yeast cellcycle regulated genes [33] and the human immune response to inflammation and host response to injury [8] both for coherent values and behaviors of the genes (MSR and LSL evaluation functions respectively). An early version of TriGen was presented in [14]. The algorithm has been improved by adding a new correlated based evaluation function $f_{L S L}$. It has been re-executed on the already used datasets, providing new improved results, and a new dataset related to human inflammation and host response to injury has been used. A validation step has been added to the methodology, based on the Gene Ontology project, providing functional annotations for the genes.

The rest of the paper is structured as follows. A review of the latest related works can be found in Section 2. Section 3 describes the methodology of the TriGen algorithm, including a detailed description of the genetic operators used and the two fitness functions. In Section 4, we show the results of applying TriGen to the synthetic dataset, the yeast cell cycle and the inflammation and the host response to injury problems. Section 5 shows the conclusions.

\section{Related works}

This section is to provide a general overview of recent works in the field of gene expression time series data. In particular, those focused on the application of triclustering. Despite the vast variety of (bi)clustering methods existing, there is no one apparently capable of extracting meaningful information from a wide range of gene expression temporal data. On the contrary, the use of triclustering seems to be the most suitable strategy due to its ability to deal with the third dimension: The time.

In 2005, Zhao and Zaki [41] introduced the triCluster algorithm to extract patterns in 3D gene expression data. They also presented a set of metrics to assess its quality and tested the approach on real microarrays. An extended and generalized version (g-triCluster) was published one year later [18]. The generalization claimed by the authors was based on the discovery of more coherent triclusters and on its robustness to noise.

Although this algorithm was able to successfully discover such patterns, the authors in [1] addressed the existing problem associated with its NP-completeness. To overcome this drawback, they proposed a parallelized version using the filter-labeledstream paradigm, exhibiting great improvement in terms of computational cost.

A new definition for coherent triclusters focused on finding regulatory relationships among genes can be found in [40]. The algorithm was applied on both synthetic and real data. Later on [38], the authors proposed an enhanced model to retrieve timedelayed clusters. The novelty lied on the discovery of gene expression cycle time, essential task to create gene regulatory networks.

Another approach, LagMiner, was introduced in [39] to find time-lagged 3D clusters. The highlighting feature claimed by the authors was, this time, its ability to discover triclusters satisfying the constraints of coherence, regulation, minimum gene number, sample subspace size and time period length. As for previous works, it was evaluated on both synthetic and real-life datasets.

The evolutionary computation has also been used in the search for triclusters. Particularly, a multi-objective algorithm that simultaneously optimizes several conflicting requirements was presented in [19]. Again, the algorithm was tested on real datasets.

A new strategy to mine 3D-clusters in real-valued data was introduced in [32]. In particular, the authors were concerned about discovering subspaces with a significant number of items, one of the main drawbacks typically found in tricluster-based approaches. The authors applied its methodology to a large number of synthetic datasets and, additionally, to a real-world case study. At the same conference, another approach focused on a triclustering method to mine quantitative data was presented [17]. However, the main concern of the authors was, this time, to discover triclusters with low variance. Their approach was successfully tested on a synthetic dataset and on a cross-species genomic dataset, yielding new insights on this topic.

The work in [20] introduced a tricluster-based approach to discover temporal dependency association rules in microarray datasets. The rules obtained are to represent regulated relations among genes.

Finally, a brief survey on triclustering applied to gene expression time series was published in 2011 [22]. It provides a good starting point for those researchers novel in this topic.

\section{Methodology}

In this section, we describe the implementation of the TriGen algorithm, our proposal to mine three dimension gene expression data. The evolutionary process is shown in detail: all the operators involved as well as the fitness functions used on the evaluation step.

A tricluster TC is as a subset from a dataset $D$ which contains information related to the behavior of some genes $G_{D}$ at a set of times $T_{D}$ under a set of conditions $C_{D}$. The tricluster $T C$ is formally defined as $T C=T \times C \times G$ where $T \subseteq T_{D}, C \subseteq C_{D}$ and $G \subseteq G_{D}$. Qualitatively, a tricluster $T C$ will provide information on the pattern of behavior of a subset of genes under certain conditions and at certain time points.

TriGen is based on a genetic algorithm. This evolutionary process has several steps (see Fig. 1): an initialization step in which the initial population will be created taking into account overlapping with previously found triclusters; an evaluation step in which the quality of each individual will be measured; a selection step, which serves to decide which individuals will survive to the next generation; 


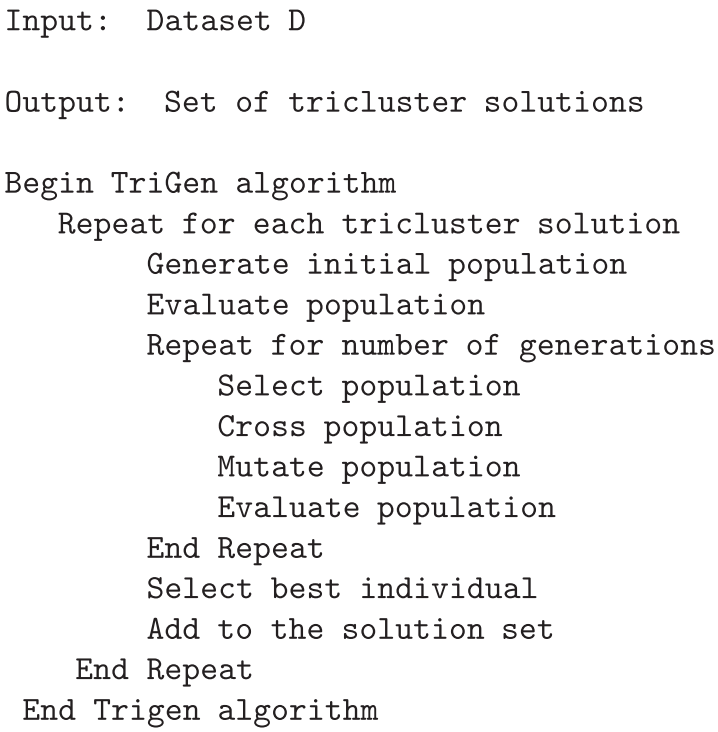

Fig. 1. TriGen algorithm.

crossover, which creates the necessary connections between pairs of individuals to share new genetic material and, finally, mutation, which performs punctual changes to individuals to ensure genetic variability of future generations. We now describe each of these operators.

\subsection{Codification of individuals}

Each individual of the population represents a tricluster $T C$ which is a potential solution. It contains genetic material that will be manipulated by the genetic operators described below. The genetic material is structured as follows. An individual is composed of three sequences of structures: one for the sequence of genes $G$ from the input dataset $D$, one for the sequence of conditions $C$, and one sequence of time points $T$. These sequences are set up based on the input matrix, that is

$G=\left\langle g_{i_{1}}, g_{i_{2}}, \ldots, g_{i_{B}}\right\rangle$

where $B$ is the number of genes listed in the input matrix, $i_{j}<i_{j+1}$ for all genes, and $1<i_{j}<B$.

Analogously:

$C=\left\langle c_{i_{1}}, c_{i_{2}}, \ldots, c_{i_{L}}\right\rangle$

where $L$ is the number of conditions listed in the input matrix, $i_{j}<i_{j+1}$ for all conditions, and $1<i_{j}<L$.

Finally, $T$ represents different time stamps or values of pairs gene-condition at different times:

$T=\left\langle t_{i_{1}}, t_{i_{2}}, \ldots, t_{i_{M}}\right\rangle$

where $M$ is the number of samples measured over time, and $t_{i_{1}}<t_{i_{2}}<\cdots<t_{i_{M}}$.

The algorithm's population is made up of several individuals, as depicted in Fig. 2.

\subsection{Generation of initial population}

The initial population is randomly generated. Part of the individuals are purely random generated, this is, a random subset of genes, conditions and times $T C=T \times C \times G$ are assigned. The rest of the individuals are also randomly generated but attending to some considerations to promote visiting of non-explored areas and visiting the widest area possible of the dataset $D$. For this purpose, each time a new solution tricluster TC is generated, the

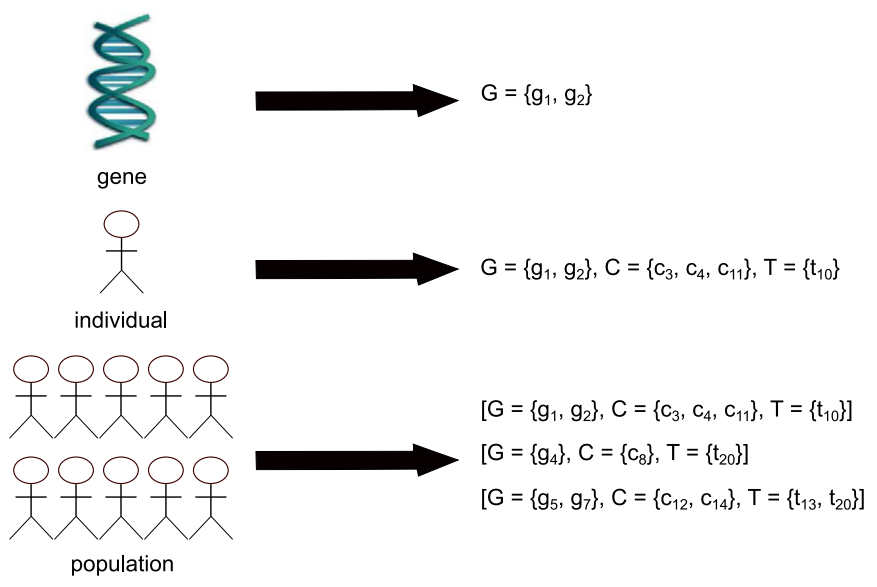

Fig. 2. Genetic algorithm codification.

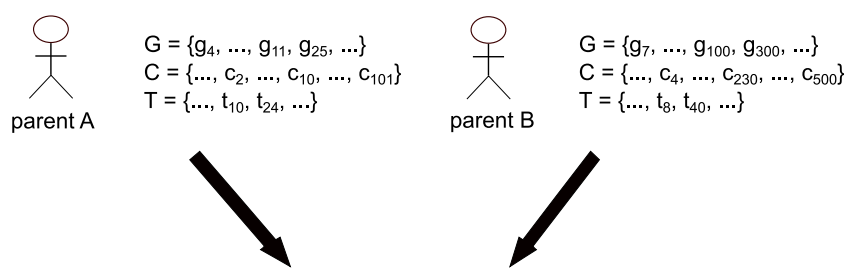

For each subset of coordinates G, C and T select a random cross point and combine

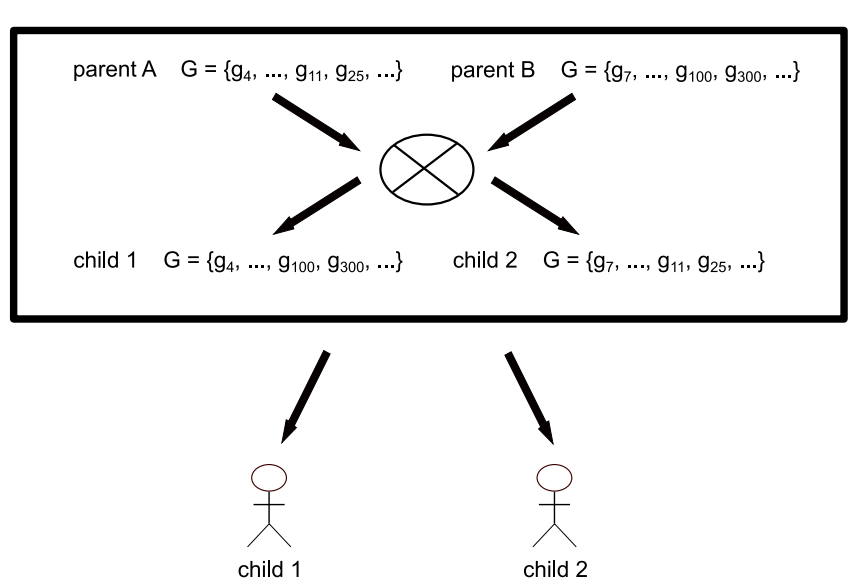

Fig. 3. Representation of the crossover operator.

coordinates of its genes $G$, conditions $C$ and times $T$ are stored. These new individuals will be created by a random subset $T \times C \times$ $G$ which had not appeared in the previous solutions.

\subsection{Crossover operator}

Two individuals (parents, $P_{1}$ and $P_{2}$ ) are combined to create two new individuals (offsprings, $O_{1}$ and $O_{2}$ ). They are chosen based on a probability of crossover parameter, denoted by $p_{c}$. Their genetic material is combined by a random one-point cross in the genes $G$, conditions $C$ and times $T$ and mixing the coordinates in both children.

Formally, let be $P_{1}{ }^{i}$ and $P_{2}{ }^{i}$ parents \#1 and \#2 at iteration $i$. The resulting offspring at this iteration is

$\left\{O_{1}^{i}, O_{2}^{i}\right\}=\tilde{\mathfrak{f}}_{C, G, T}\left(P_{1}^{i}, P_{2}^{i}\right)$

where $\mathfrak{f}$ is the function than randomly selects a subset of $(C, G, T)$ from parents $P_{1}{ }^{i}$ and $P_{2}{ }^{i}$ at iteration $i$, given a probability of crossover $p_{c}{ }^{i}$. The whole procedure is illustrated in Fig. 3. 
The procedure can be formalized as follows. Let $S_{1}=\left\langle g_{i_{1}}, \ldots, g_{i_{m}}\right\rangle$ and $S_{2}=\left\langle g_{j_{1}}^{\prime}, \ldots, g_{j_{n}}^{\prime}\right\rangle$ be two sequences of genes, where $g_{i_{k}}, g_{j_{l}} \in G$, $i_{1}<\cdots<i_{m}, j_{1}<\cdots<j_{n}$ and $m, n<B$.

Let $p$ be a number randomly chosen from 1 and $\min (m, n)$. The new two individuals or children are formed as follows:

$O_{1}=\left\langle g_{i_{1}}, \ldots, g_{i_{p}}, g_{j_{p+1}}^{\prime}, \ldots, g_{j_{n}}^{\prime}\right\rangle$

$O_{2}=\left\langle g_{j_{1}}^{\prime}, \ldots, g_{j_{p}}^{\prime}, g_{i_{p+1}}, \ldots, g_{i_{m}}\right\rangle$

Note that after the creation of both offsprings, the elements must be reordered since positions $j_{l}$ may not be necessarily greater than positions $i_{k}$ and viceversa. Additionally, both $S_{1}$ and $S_{2}$ may share any gene, as they are randomly formed from the input matrix. Should it be the case, repeated elements must be removed from $O_{1}$ and $O_{2}$. Therefore, the number of genes composing $O_{1}$ may eventually be less than $n$ and the number of genes in $\mathrm{O}_{2}$ could be less than $m$.

The full process is analogously performed for conditions and time stamps.

\subsection{Mutation operator}

An individual can be mutated according to a probability of mutation, $p_{m}$. The $p_{m}$ condition is verified for every individual and if it is satisfied, one out of six possible actions is taken. These actions are: add a new random gene coordinate to $G$, add a new condition coordinate to $C$ or add a new time coordinate to $T$, or by removing a random coordinate from either $G, C$ or $T$. The election of these actions is also random. For the case of addition of new coordinates, it is first checked that the new randomly selected coordinate is not included yet in $G, C$ or $T$. If so, the process is repeated until finding a new coordinate that did not originally belong to the individual. Again, the sequence must be reordered so that the new sequence remains ordered.

\subsection{Selection operator}

This operator is implemented following the roulette wheel selection method [23]. The fitness level is used to associate a probability of selection with each individual of the population. This emulates the behavior of a roulette wheel in a casino. Usually a proportion of the wheel is assigned to each of the possible selections based on their fitness value. Then a random selection is made similar to how the roulette wheel is rotated. While candidates with a higher fitness will be less likely to be eliminated, there is still a chance that they might be. There is a chance that some weaker solutions may survive the selection process, which is an advantage, as though a solution may be weak, it may include some component which could prove useful following the recombination process.

\subsection{Fitness function}

The fitness of each individual allows the algorithm to determine which are the best candidates to remain in subsequent generations. For the TriGen algorithm, we have implemented two different fitness functions, the first one based on a three dimensions adaptation of the Mean Square Residue measure (MSR) which is a classic biclustering measure for gene expression analysis [9], from now on referred as $f_{M S R}$. The second one $f_{L S L}$. The first function $f_{M S R}$ is defined based on a distance function, while $f_{L S L}$ is more suitable to find correlations among a set of genes even if they are expressed at different levels of magnitude.

Both functions share some common terms which are now defined.
- G: Subset of gene coordinates of the individual.

- $C$ : Subset of condition coordinates of the individual.

- T: Subset of time coordinates of the individual.

- $T_{l}$ : Number of time coordinates of the individual.

- $C_{l}$ : Number of condition coordinates of the individual.

- $G_{l}$ : Number of gene coordinates of the individual.

- $T C_{v}(t, g, c)$ : Expression level of gene $g$ under condition $c$ at time $t$ as seen in the input dataset $D$.

Weights term 1. The Weights term is defined as

Weights $=G_{l} * w_{g}+C_{l} * w_{c}+T_{l} * w_{t}$

where $w_{g}, w_{c}$ and $w_{t}$ are weights for the number of genes, conditions and times in a tricluster solution $T C$, respectively. With high values of the weights, we favor that TriGen finds solutions with many components for that term.

Distinction term 1. The Distinction term is defined as

$$
\begin{aligned}
\text { Distinction }= & \frac{C D N_{g}}{G_{l}} * w d_{g}+\frac{C D N_{c}}{C_{l}} * w d_{c} \\
& +\frac{C D N_{t}}{T_{l}} * w d_{t}
\end{aligned}
$$

where $C D N_{g}$ (Coordinate Distinction Number of $g$ ), $C D N_{c}$ (Coordinate Distinction Number of $c$ ) and $C D N_{t}$ (Coordinate Distinction Number of $t$ ) are, respectively, the number of genes, conditions and time coordinates in the tricluster solutions that are not present in the tricluster being evaluated, and $w d_{g}, w d_{c}$ and $w d_{t}$ are the distinction weights of the genes, conditions and times respectively. Distinction measures how different the individual under evaluation is compared to the triclusters previously found. If the values of the weights are increased, we favor finding nonoverlapping solutions to the previously found.

\subsection{1. $f_{\text {MSR }}$ fitness function}

This function is defined by the following equation:

$f_{\text {MSR }}(T C)=$ MSR - Weights - Distinction

It is a minimizing function (the smaller the value the better) which has three terms, the general Weigths and Distinction terms and the specific MSR term. The MSR term can be explained as follows.

MSR term 1. Since triclustering emerges as an improvement of biclustering to analyze microarray data taking into account the temporal dimension, we have adapted a classical biclustering fitness function, Mean Squared Residue (MSR), presented by Cheng and Church in [9], to the three dimensional space. MSR compares the similarity of each value in the bicluster to the mean values of all genes under the same condition, the mean of the gene under the other conditions included in the bicluster, and the mean of all values in the bicluster. In the case of triclustering, we will assess the similarity of each value not only related to genes and conditions, but also including the temporary plane, i.e., we asses how a gene $g$ behaves under all conditions $C$ at the time points $T$, how a condition $c$ affects all genes $G$ in time $T$, and the time factor $t$ in relation to genes $G$ and conditions $C$, as well as the mean value of all the tricluster. This is formalized as follows:

$M S R=\frac{\sum_{g \in G, c \in \in C, t \in T} r_{g c t}^{2}}{G_{l} * C_{l} * T_{l}}$

where $r_{\text {gct }}$ can be defined as

$r_{g c t}=T C_{v}(t, g, c)+M_{G C}(t)+M_{G T}(c)+M_{C T}(g)-M_{G}(c, t)$

$-M_{C}(g, t)-M_{T}(g, c)-M_{G C T}$

where $M_{G C}(t)$ is the mean of the genes under conditions at a point in time $t, M_{G T}(c)$ is the mean of the genes over time under 

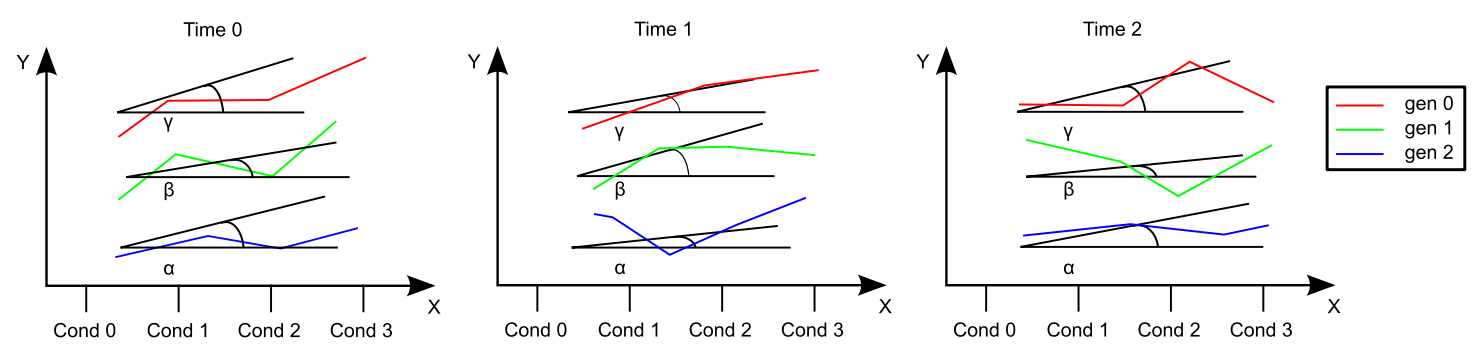

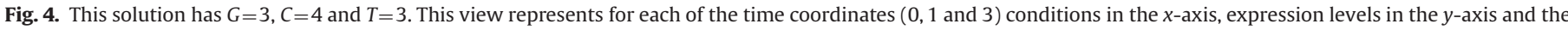

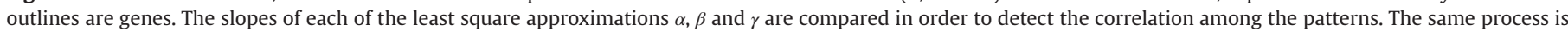
followed with the other views.

a condition $c, M_{C T}(g)$ is the mean of a gene $g$ in time under the conditions, $M_{G}(c, t)$ is the mean of the genes under one condition and a time point, $M_{C}(g, t)$ is the mean of the values of a gene at a time point under conditions, $M_{T}(g, c)$ is the mean of a gene under a condition at all time points and $M_{G C T}$ is the mean value of all values in the tricluster.

\subsection{2. $f_{L S L}$ Fitness function}

This function is defined by the following equation:

$f_{L S L}(T C)=L S L-$ Weights - Distinction

It is a minimizing function (the smaller the value the better) which has three terms, the general Weigths and Distinction terms and the specific $L S L$ term, explained as follows.

LSL term 1. This term is defined by Eq. (13).

$L S L=\frac{T_{r}+C_{r}+G_{r}}{3}$

It measures the similitude between the least squares approximation for the points in each graphic of the three views that represent a tricluster: first, for each time coordinate, conditions in the $\mathrm{x}$-axis, expression levels in the $\mathrm{y}$-axis and the outlines are genes $\left(T_{r}\right.$ in (13)); second, for each condition coordinate, times in the $x$-axis, expression levels in the $y$-axis and the outlines are genes $\left(C_{r}\right.$ in (13)); and third, for each condition coordinate, genes in the $x$-axis, expression levels in the $y$-axis and the outlines are times $\left(G_{r}\right.$ in (13)). We can see a representation of this in Fig. 4.

All elements in the numerator in Eq. (13) have in common the values indicated in equation group (14).

$\operatorname{sum} X_{t c}=\sum_{g \varepsilon G} g \quad \operatorname{sumXX} X_{t c}=\sum_{g \varepsilon G} g^{2}$

$\operatorname{sum}_{g}=\sum_{t \varepsilon T} t \quad \operatorname{sumXX}=\sum_{t \varepsilon T} t^{2}$

where $\operatorname{sum} X_{t c}$ is the summation of all genes of the individual under evaluation, sumXX $X_{t c}$ is the squared summation of all genes, sum $X_{g}$ is the summation of all times and $\operatorname{sumXX}$ is the is the squared summation of al times.

As you can see in Eq. (15a), $T_{r}$ is the distance between all least square approximations in the first view described above.

$T_{r}=\frac{\sum_{t_{i}, t_{j} \varepsilon T}\left|T D_{t_{i}}-T D_{t_{j}}\right|}{\left(T_{l}-1\right) * T_{l}}$

$\forall t \in T, \quad T D_{t}=\frac{G_{l} * \operatorname{sumX} Y_{t}-\left(\operatorname{sum} X_{t c} * \operatorname{sum} Y_{t}\right)}{G_{l} * \operatorname{sumX} X X_{t c}-\operatorname{sum} X_{t c}^{2}}$

$\forall t \in T, \quad \operatorname{sumXY} Y_{t}=\sum_{g \varepsilon G c \varepsilon C} \sum_{i} g * I N_{v}(t, g, c)$

$\forall t \in T, \quad \operatorname{sum} Y_{t}=\sum_{g \varepsilon G c \varepsilon C} \sum_{V} N_{v}(t, g, c)$ where measures are represented by TD (15b) and $\operatorname{sumXY} Y_{t}$ and sum $_{t}$ are, respectively, for each individual time, the summation of each expression level value of this time and all combinations of individual genes and conditions multiply by the genes (15c) and for each individual time, the summation of each expression level value of this time and all combinations of individual genes and conditions (15d).

In the same way, Eq. (16a) defines $C_{r}$ term as the distance between all least square approximations produced in the second view.

$C_{r}=\frac{\sum_{c_{i}, c_{j} \varepsilon C}\left|C D_{c_{i}}-C D_{c_{j}}\right|}{\left(C_{l}-1\right) * C_{l}}$

$\forall c \in C, \quad C D_{c}=\frac{G_{l} * \operatorname{sum} X Y_{c}-\left(\operatorname{sum} X_{t c} * \operatorname{sum} Y_{c}\right)}{G_{l} * \operatorname{sum} X X_{t c}-\operatorname{sum} X_{t c}^{2}}$

$\forall c \in C, \quad \operatorname{sumXY}_{c}=\sum_{t \varepsilon T g_{\varepsilon} G} g * I N_{v}(t, g, c)$

$\forall c \in C, \quad \operatorname{sum}_{c}=\sum_{t \varepsilon T} \sum_{g \varepsilon G} I N_{v}(t, g, c)$

where measures are represented by $C D(16 \mathrm{~b})$ and $\operatorname{sumXY}_{c}$ and $\operatorname{sum}_{c}$ are, respectively, for each individual condition, the summation of each expression level value of this condition and all combinations of individual times and genes multiply by the genes (16c) and for each individual condition, the summation of each expression level value of this condition and all combinations of individual times and genes (16d).

Finally, Eq. (17a) defines the $G_{r}$ term as the distance between all least square approximations produced in the third view described above.

$G_{r}=\frac{\sum_{c_{i}, c_{j} \varepsilon}\left|G D_{c_{i}}-G D_{c_{j}}\right|}{\left(C_{l}-1\right) * C_{l}}$

$\forall c \in C, \quad G D_{c}=\frac{T_{l} * \operatorname{sum} X Y_{g}-\left(\operatorname{sum} X_{g} * \operatorname{sum} Y_{g}\right)}{T_{l} * \operatorname{sumX} X_{g}-\operatorname{sum} X_{g}^{2}}$

$\forall c \in C, \quad \operatorname{sumXY}_{c}=\sum_{g \varepsilon G t \varepsilon T} t * I N_{v}(t, g, c)$

$\forall c \in C, \quad \operatorname{sum}_{c}=\sum_{g_{\varepsilon} G t \varepsilon T} \sum_{t} I N_{v}(t, g, c)$

where measures are represented by GD (17b) and $s u m X Y_{c}$ and $\operatorname{sum}_{c}$ are, respectively, for each individual condition, the summation of each expression level value of this condition and all combinations of individual genes and times multiply by the times (17c) and for each individual condition, the summation of each expression level value of this condition and all combinations of individual genes and times (17d). 


\section{Results}

We show the results obtained applying the TriGen algorithm both to real and synthetic data. Synthetic data has the advantage that the process that generates the data is well known and so one is able to judge the success or failure of the algorithm [24]. Synthetic datasets generation has been widely applied both in microarray related publications $[5,15]$, and in other general data mining applications [26].

TriGen takes several parameters: probability of crossover $p_{c}$, probability of mutation $p_{m}$, weights $w_{g}$ for the number of genes, $w_{c}$ for the conditions and $w_{t}$ for the times, used to calculate the term Weights (see Section 3.6), and weights $w d_{g}, w d_{c}$ and $w d_{t}$ are the distinction weights of the genes, conditions and times respectively, used to calculate the term Distinction (see Section 3.6).

All experiments were executed on a multiprocessor machine with 64 processors Intel Xeon E7-4820 $2.00 \mathrm{GHz}$ with 8 GB RAM memory. We now describe the results obtained.

\subsection{Results on the synthetic dataset}

The set of synthetic data has been generated using a software application developed for such purpose. For this particular work, we have simulated data from 5 different time points and 10 conditions using microarrays containing 1000 genes. Each gene is assigned a value which represents its level of expression, i.e., quantification of the mRNA present in a time point under certain conditions. This value has been randomly chosen from the rank, respectively for each condition, [1, 15], [7,35], [60,75], [0,25), $[30,100],[71,135],[160,375],[5,30],[25,40]$ and $[10,30]$. In such data set, we have allocated two areas ( $\mathrm{a}$ and $\mathrm{b}$ ) of prefixed values:

Area a: It is an area of size $G_{l}=20, C_{l}=5$ and $T_{l}=3$ with all its values fixed to 1 .

Area b: It is an area with $G_{l}=30, C_{l}=4$ and $T_{l}=4$ with an ascending pattern at times $t=0,1$ and descending at times $t=2,3$ at different levels of magnitude $[1,15]$, [60, 75], [5, 30] and [160, 375].

TriGen was executed with each of the fitness functions available, $f_{M S R}$ and $f_{L S L}$ with the parameters in Table 1 . The weights $w_{c}$ and $w_{t}$ are high in relation to $w_{g}$ since the genes show high dimensionality in relation to conditions and times. The distinction is not taken into account in these executions. The TriGen algorithm did successfully find areas $a$ and $b$ in both executions. The execution with fitness function $f_{M S R}$ found the whole area $a$ and missed some genes (seven out of thirty) and missed one time point (three out of four). The execution with $f_{L S L}$ found tricluster solutions for both areas $a$ and $b$. This might be due to the fact that area $a$, with fixed values can be easily detected both by distance

Table 1

Parameters for the execution of the TriGen algorithm on the synthetic dataset.

\begin{tabular}{lc}
\hline Parameter & Value \\
\hline Number of solutions & 4 \\
Generations & 50 \\
Members in the population & 100 \\
$p_{c}$ & 0.7 \\
$p_{m}$ & 0.3 \\
$w_{g}$ & 0.01 \\
$w_{c}$ & 0.55 \\
$w_{t}$ & 0.35 \\
$w d_{g}$ & 0.0 \\
$w d_{c}$ & 0.0 \\
$w d_{t}$ & 0.0 \\
\hline
\end{tabular}

and correlation measures. In contrast, area $b$ exhibits correlation patterns, and therefore is easily found by $f_{L S L}$ and $f_{M S R}$ was capable of only finding part of it.

\subsection{Results on the yeast dataset}

We have applied the TriGen algorithm to the yeast (Saccharomyces cerevisiae) cell cycle problem [33]. The yeast cell cycle analysis project's goal is to identify all genes whose mRNA levels are regulated by the cell cycle. By applying TriGen to this dataset, we aim at finding meaningful patterns on this cell cycle. In this experiment, 6179 genes are analyzed under 6 conditions, termed cln3, clb2, pheromone, cdc15, cdc28 and elutriation [33]. Samples were taken at 2 time points for $\operatorname{cln} 3,2$ for clb2, 18 for pheromone, 24 for cdc15, 17 for cdc28 and 14 for elutriation. To apply the TriGen algorithm we did not take into account the conditions with only 2 time points and we used the first 14 time points of the pheromone, cdc15, cdc28 and elutriation experiment, in order to have a compact dataset. Therefore our dataset contains 6179 genes, 4 conditions and 14 time points.

For this experiment, after some preliminary executions, we chose the set of parameters in Table 2 and executed both with the $f_{M S R}$ fitness function and with the $f_{L S L}$. The value of $w_{g}$ was set to 0.8 favor the presence of several genes in the solutions, since the algorithm had a tendency to provide solutions with a low number of genes. This might be due to the fact that the genes do not exhibit exactly equal patterns, neither for the distance nor the correlation measure. For the parameters related to the Distinction term we provide a high value for the genes in order to cover as much space in this dimension as possible. We also provide two measures to better understand the results obtained: overlapping of the 20 triclusters obtained, with a value of 0.1 , this value due to the overlapping of conditions and times, which appear repeated in the solutions due to their low dimensionality, and coverage of the solutions, which represents the proportion of cells from the input dataset $D$ present in the solutions.

We were interested in the relation among the 20 triclusters generated by $f_{M S R}$ and the 20 generated by $f_{L S L}$ (see Table 2). We calculated the average overlapping of the two groups, comparing each tricluster generated by $f_{M S R}$ to each tricluster generated by $f_{L S L}$. On average, the overlapping of the genes is as low as $0.07 \%$, while the overlapping of conditions is $75 \%$ and the overlapping of time points is $53 \%$. The percentages agree with the number of potential genes, conditions and time points for each tricluster, 6179, 6 and 14 respectively.

For legibility reasons, we focus on one of the solutions. It was obtained with the $f_{M S R}$ measure. It groups 20 genes under 2 conditions, pheromone (condition 0 ) and cdc28 (condition 3 ) and five

Table 2

Parameters for the execution of the TriGen algorithm on the yeast cell cycle dataset.

\begin{tabular}{lc}
\hline Parameter & Value \\
\hline Number of solutions & 20 \\
Generations & 400 \\
Members in the population & 200 \\
$p_{c}$ & 0.8 \\
$p_{m}$ & 0.5 \\
$w_{g}$ & 0.8 \\
$w_{c}$ & 0.1 \\
$w_{t}$ & 0.1 \\
$w d_{g}$ & 1.0 \\
$w d_{c}$ & 0.0 \\
$w d_{t}$ & 0.0 \\
Overlapping & 0.1 \\
Coverage & 0.08 \\
\hline
\end{tabular}


a

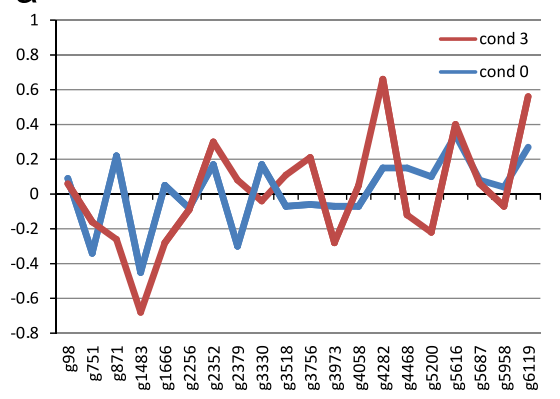

b

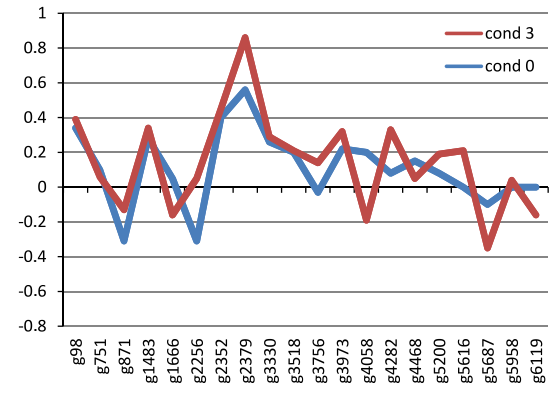

C

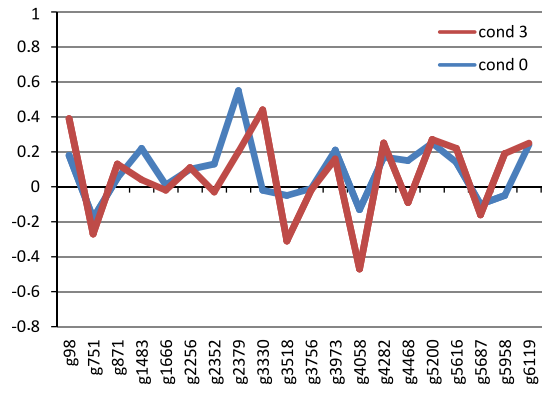

d

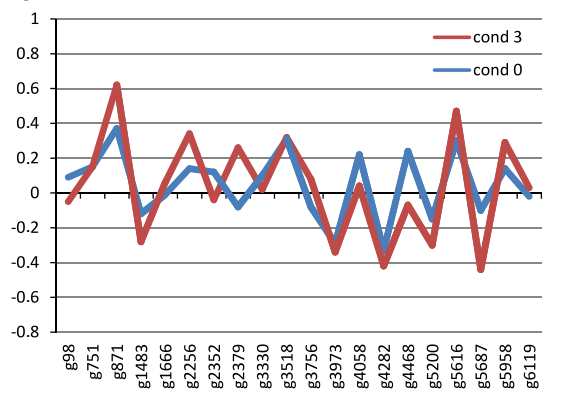

e

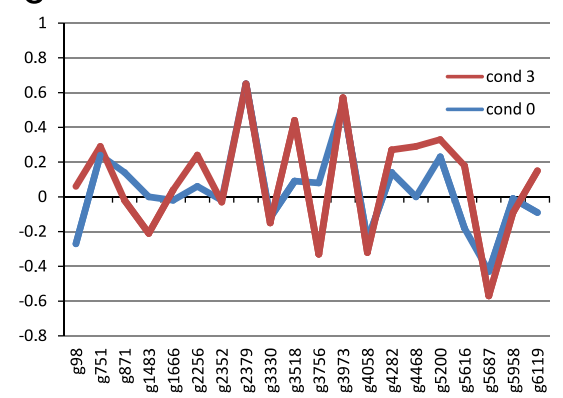

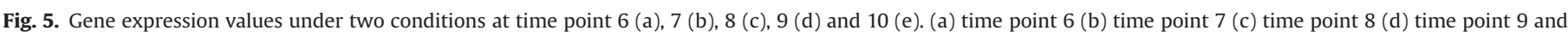
(e) time point 10 .

a

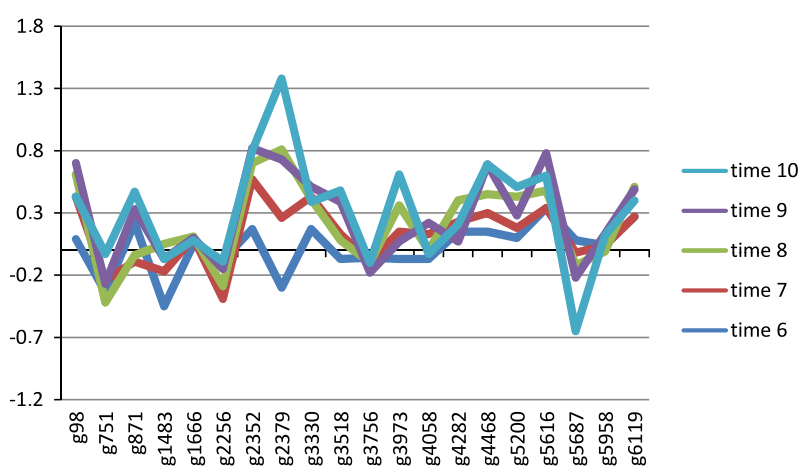

b

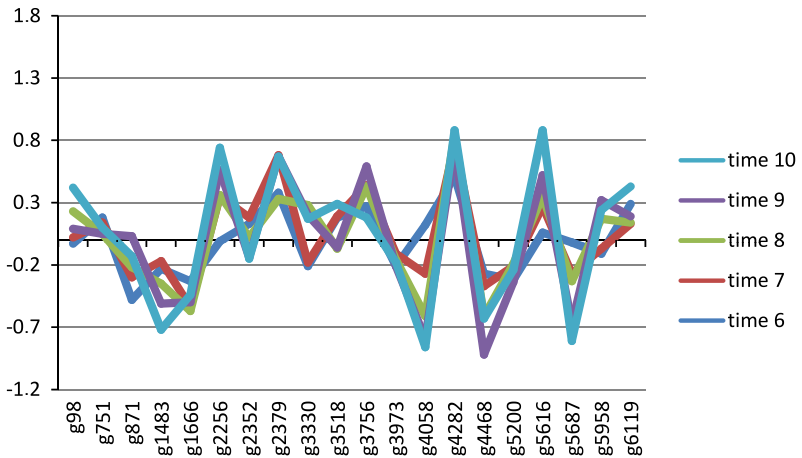

Fig. 6. Gene expression values under five time points at pheromone (a) and cdc28 (b) experiments. (a) condition 0 and (b) condition 3.

time points $(6,7,8,9$ and 10$)$. The graphics are organized in three groups related to this solution: in Fig. 5 we present the outline of the gene expression values ( $y$-axis) for each gene in the solution $(x-$ axis) comparing the pheromone (condition 0 ) and cdc28 (condition 3). A graphic is provided for each time point: 6(a), 7(b), 8(c), 9 (d) and 10(e). In Fig. 6, we present the outline of gene expression
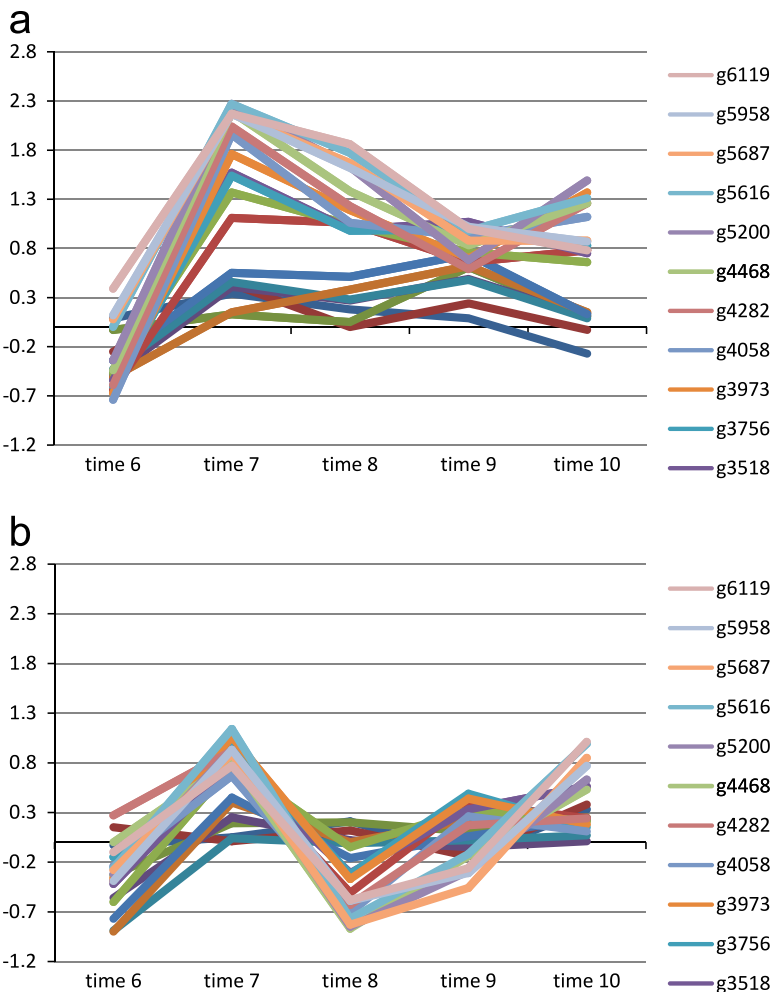

Fig. 7. Gene expression for five time points under gene solution set at pheromone (a) and cdc28 (b) experiments. (a) condition 0 and (b) condition 3.

values ( $y$-axis) for each gene in the solution ( $x$-axis) comparing time points $6,7,8,9$ and 10 and providing a graphic for each condition in the solutions: pheromone (a) and cdc28 (b). Finally in Fig. 7 we present the outline of gene expression values ( $y$-axis) for each time point ( $x$-axis) providing a graphic for each condition: pheromone (a) and cdc28 (b). As we see, in the three dimensions depicted the 
Table 3

GO for yeast cell cycle results.

\begin{tabular}{|c|c|c|c|c|}
\hline $\begin{array}{l}\text { Cluster } \\
\text { ID }\end{array}$ & Biological process & Molecular function & Cellular component & PI \\
\hline 19 & & $\begin{array}{l}\text { Pyruvate dehydrogenase (lipoamide) } \\
\text { phosphatase activity }\end{array}$ & Actin cap & $8.54 \mathrm{E}-05$ \\
\hline 2 & & Endopeptidase activity & $\begin{array}{l}\text { Vacuolar lumen (sensu } \\
\text { Fungi) }\end{array}$ & $1.18 \mathrm{E}-03$ \\
\hline 14 & Protein metabolism, cytokinesis & & & $1.79 \mathrm{E}-03$ \\
\hline 25 & Protein metabolism & & Nucleus & $2.47 \mathrm{E}-03$ \\
\hline 9 & Cytokinesis, regulation of biological process & & & $5.64 \mathrm{E}-03$ \\
\hline 23 & & $\begin{array}{l}\text { Transcription regulator activity, } \\
\text { binding, catalytic activity }\end{array}$ & Intracellular & $1.54 \mathrm{E}-02$ \\
\hline 12 & Oligosaccharide-lipid intermediate assembly & & Cytoplasm & $1.71 \mathrm{E}-02$ \\
\hline 21 & Organelle organization and biogenesis, cell proliferation & & $\begin{array}{l}\text { Intracellular membrane- } \\
\text { bound organelle }\end{array}$ & $1.72 \mathrm{E}-02$ \\
\hline 11 & & Signal transducer activity & Intracellular, Organelle & $1.96 \mathrm{E}-02$ \\
\hline 31 & Cytokinesis, cellular morphogenesis & Structural constituent of cytoskeleton & & $1.96 \mathrm{E}-02$ \\
\hline 22 & Protein complex assembly, cell proliferation & & Nucleus, protein complex & $2.60 \mathrm{E}-02$ \\
\hline 30 & Ribosome biogenesis and assembly, metabolism & Transporter activity & Cytoplasm, nucleus & $2.68 \mathrm{E}-02$ \\
\hline 6 & & Copper ion binding & & $2.93 \mathrm{E}-02$ \\
\hline 16 & Protein amino acid dephosphorylation & & Cytoplasm & $3.52 \mathrm{E}-02$ \\
\hline 13 & Regulation of biological process & & $\begin{array}{l}\text { Cell wall (sensu Fungi), } \\
\text { intracellular }\end{array}$ & $3.5 \mathrm{E}-02$ \\
\hline 34 & Cellular process, protein biosynthesis & & Cytoplasm & $3.76 \mathrm{E}-02$ \\
\hline 10 & $\begin{array}{l}\text { Vacuole fusion, non-autophagic, regulation of phosphate metabolism, signal } \\
\text { transduction, pseudohyphal growth }\end{array}$ & & Nucleus & $3.99 \mathrm{E}-02$ \\
\hline
\end{tabular}

behavior of the genes is very similar throughout the conditions and the times.

We provide a validation of the solutions obtained based on the Gene Ontology project (GO) [2]. GO is a major bioinformatics initiative with the aim of standardizing the representation of gene and gene product attributes across species and databases. The project provides an ontology of terms for describing gene product characteristics and gene product annotation data. The ontology covers three domains: cellular component, the parts of a cell or its extracellular environment; molecular function, the elemental activities of a gene product at the molecular level, such as binding or catalysis; and biological process, operations or sets of molecular events with a defined beginning and end, pertinent to the functioning of integrated living units: cells, tissues, organs, and organisms. We have queried the terms associated to our genes in GO using the Onto-CC tool [29,28], an automatic method specially suited for independent validation of gene grouping hypotheses (e.g. co-expressed genes) based on GO clusters (i.e. expression versus GO). Onto-CC reduces the uncertainty of the queries by identifying optimal conceptual clusters that combine terms from different ontologies simultaneously, as well as terms defined at different levels of specificity in the GO hierarchy. A probability of intersection $p$-value is given for each group of genes returned by Onto-CC. This value lies in the [0-1] interval and groups of genes are considered as relevant with values below 0.05 . In Table 3, we show the validation results using the Onto-CC program for this tricluster solution. The $p$-values obtained are quite low (up to $8.54 \mathrm{E}-05$ ) and the terms provided are very specific and many of them directly related to the cell cycle such as those in cluster IDs 2 , 9, 10 and 21.

For further validation of the solutions provided by TriGen, we have executed the Onto-CC tool with ten groups of genes randomly chosen from the 6179 genes in the yeast dataset, each group containing 20 genes as in the tested solution. The best (minimum) $p$-value obtained has been 0.025 , and the deepest level in the ontologies has been 3 , very generic terms. Therefore, we can conclude that the genes selected by TriGen have a closer relation in terms of GO annotations than expected if they were selected at random.
Table 4

Parameters for the execution of the TriGen algorithm on the inflammation and host response to injury dataset.

\begin{tabular}{lc}
\hline Parameter & Value \\
\hline Number of solutions & 20 \\
Generations & 100 \\
Members in the population & 200 \\
$p_{c}$ & 0.7 \\
$p_{m}$ & 0.4 \\
$w_{g}$ & 0.9 \\
$w_{c}$ & 0.0 \\
$w_{t}$ & 0.1 \\
$w d_{g}$ & 0.9 \\
$w d_{c}$ & 0.0 \\
$w d_{t}$ & 0.1 \\
Overlapping & 0.08 \\
Coverage & 0.2 \\
\hline
\end{tabular}

\subsection{Results on the inflammation and host response to injury dataset}

The problem under study deals with human inflammation and the host response to injury. Understanding the inflammation process is critical because the body uses inflammation to protect itself from infection or injury (e.g., crushes, massive bleeding, or a serious burn). The host response to trauma and burns is a collection of biological and pathological processes that depends critically upon the regulation of the human immuno-inflammatory response [8].

The data has been acquired from an experiment about inflammation and host response to injury carried out with microarrays. In this experiment, blood samples from 8 volunteers are analyzed, 4 treated with a toxin that simulates an inflammatory process and 4 with a placebo. Samples were taken at 6 time points throughout $24 \mathrm{~h}$, obtaining a total of 48 microarrays. We work with a set of 2155 genes selected as relevant for the problem [30] considering 2 conditions: endotoxin and placebo.

The set of parameters chosen can be seen in Table 4 and TriGen was executed both with the $f_{M S R}$ fitness function and with the $f_{L S L}$ 
a

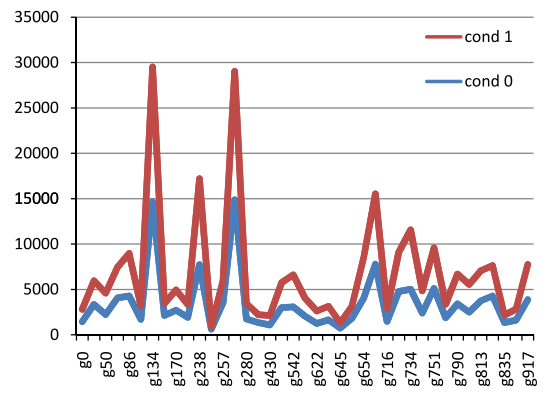

b

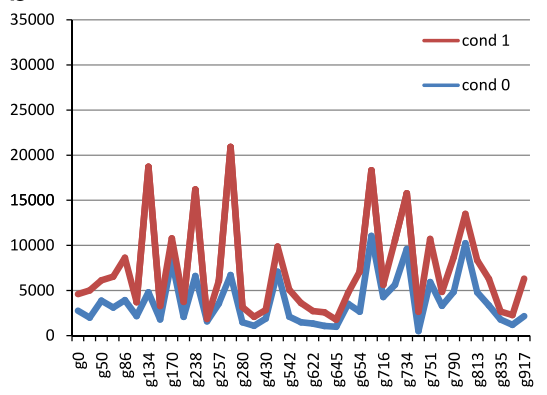

C

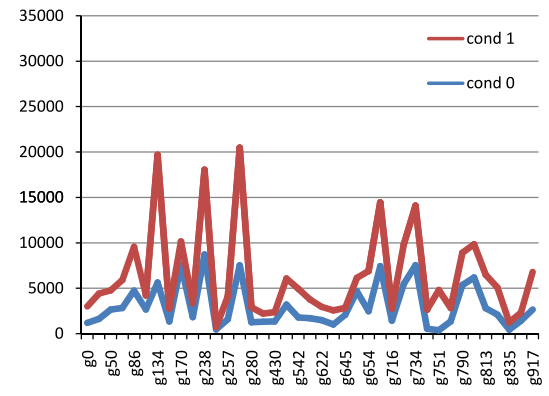

d

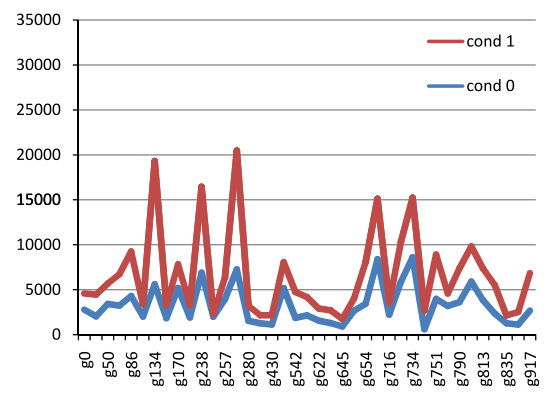

e

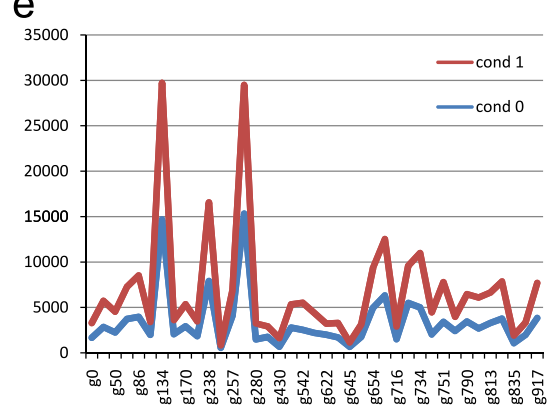

Fig. 8. Gene expression values under two conditions at time point 0 (a), 1 (b), 3 (c), 4 (d) and 5 (e).

a

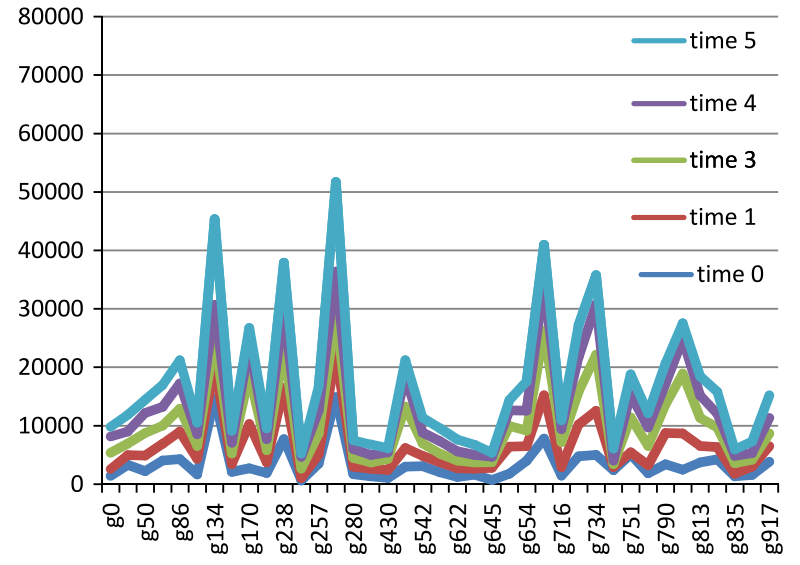

b

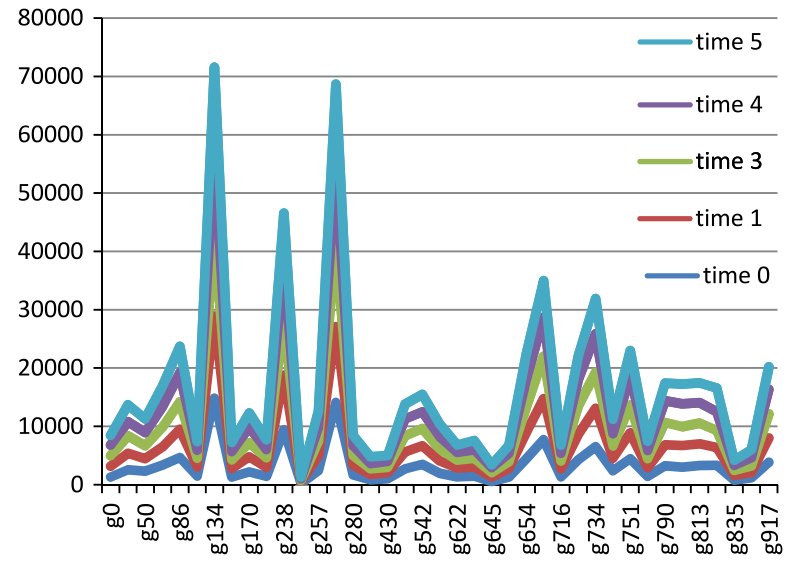

Fig. 9. Gene expression values under five time points at condition 0 (a) and condition 1 (b) experiments.

one. We can see that the number of iterations has been decreased compared to the yeast cell cycle experiment. This is due to the dimensionality of the experiments. In the yeast cell cycle problem
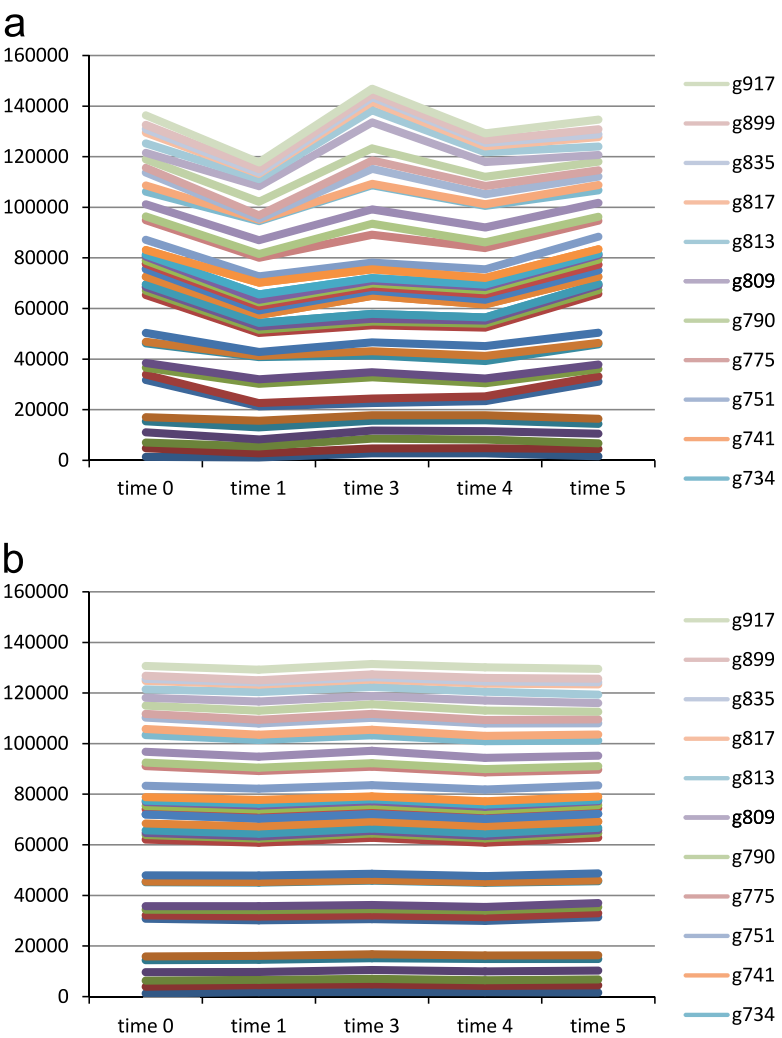

Fig. 10. Gene expression for five time points under gene solution set at condition 0 (a) and condition 1 (b) experiments.

with $G=6179, C=4$ and $T=14$ the total number of cells to visit is 346,024 . For the inflammation and host response to injury problem, $G=2155, C=2$ and $T=5$, a total number of 25,860 cells to visit. Therefore, TriGen does not need to iterate so many times. In this case, we also favor the diversity of the genes found by setting $w d_{g}$ to 0.9 . 
We also provide two measures of the solutions, overlapping of the 20 triclusters obtained, with a low value because genes, which are the ones with more influence in this measure due to the dimensionality, are very diverse. Coverage of the solutions is 0.2 from the input dataset.

We also provide information about the relation among the 20 triclusters generated by $f_{M S R}$ and the 20 generated by $f_{L S L}$ (see Table 4). We calculated the average of the overlapping of the two groups, comparing each tricluster generated by $f_{M S R}$ to each tricluster generated by $f_{L S L}$. On average, the overlapping of the genes is $1.5 \%$, while the overlapping of conditions is $100 \%$ and the overlapping of time points is $78 \%$. The percentages agree with the number of potential genes, conditions and time points for each tricluster, 2155, 2 and 6 respectively. In particular, both conditions are present in all the clusters.

Again we focus only on one of the solutions for legibility reasons. It was obtained with the $f_{L S L}$ measure. It groups 11 genes under the 2 conditions, endotoxin (condition 0 ) and placebo (condition 1) and five time points $(0,1,2,3,4,5)$.

We show three groups of graphics related to this solution: In Fig. 8, we present the outline of the gene expression values $(y$-axis) for each gene in the solution ( $x$-axis) comparing the endotoxin (condition 0 ) and placebo (condition 1). A graphic is provided for each time point: 0 (a), 1 (b), 3 (c), 4 (d) and 5 (c). In Fig. 9, we present the outline of gene expression values ( $y$-axis) for each sgene in the solution ( $x$-axis) comparing time points $0,1,3$,
4 and 5 and providing a graphic for each condition: endotoxin (a) and placebo (b). Finally, in Fig. 10 we present the outline of gene expression values ( $y$-axis) for each time point ( $x$-axis) comparing each gene in the solution providing a graphic for each condition: endotoxin (a) and placebo (b).

In this case, we see in each of the three dimensions represented the similarity of the behavior of the genes. However, we can see that the patterns exhibited are extremely correlated, much more than in the yeast cell cycle experiment. In fact, the genes are expressed at different levels of magnitude. We can see in Fig. 9 how the genes in time 0 are expressed in the $0-15,000$ interval, while in time 5 the rank in the 9000-70,000 interval. However, since those patterns are correlated they are retrieved by TriGen. This is due to the fact that this solution was found using the $f_{L S L}$ based fitness function.

We also provide the validation results using the Onto-CC program for this tricluster solution in Table 5. We see that the PI results obtained are low (up to 6.07E-09) meaning that the groups of genes are significative related to the GO terms associated to them. Furthermore, the terms are very specific as in cluster IDs $6,33,51,66$ and in particular the term immune response is present in 31 .

As for the yeast cell cycle problem, we provide further validation of the solutions provided by TriGen executing the Onto-CC tool with 10 groups of genes randomly chosen from the 2155 genes in the inflammation and host response to injury problem, each group

Table 5

GO for inflammation and host response to injury results.

\begin{tabular}{|c|c|c|c|c|}
\hline $\begin{array}{l}\text { Cluster } \\
\text { ID }\end{array}$ & Biological process & Molecular function & Cellular component & PI \\
\hline 71 & & $\begin{array}{l}\text { Cation binding, catalytic activity, transcription regulator } \\
\text { activity }\end{array}$ & & $6.07 \mathrm{E}-09$ \\
\hline 51 & Protein amino acid phosphorylation & & $\begin{array}{l}\text { Intracellular, extracellular } \\
\text { region }\end{array}$ & $6.28 \mathrm{E}-09$ \\
\hline 46 & Ion transport & ion transporter activity, protein binding & $\begin{array}{l}\text { Integral to membrane, } \\
\text { intracellular }\end{array}$ & $2.81 \mathrm{E}-07$ \\
\hline 69 & Regulation of transcription, DNA-dependent & Zinc ion binding, protein binding & & $7.66 \mathrm{E}-06$ \\
\hline 37 & Regulation of transcription, DNA-dependent & & Cytoplasm & $1.12 \mathrm{E}-05$ \\
\hline 61 & & Binding, ubiquitin-like-protein ligase activity & Cytoplasm & $1.88 \mathrm{E}-05$ \\
\hline 4 & Regulation of biological process & Binding & Intracellular & $5.12 \mathrm{E}-05$ \\
\hline 52 & $\begin{array}{l}\text { Protein metabolism, regulation of biological } \\
\text { process }\end{array}$ & Catalytic activity, nucleotide binding, nucleic acid binding & & $5.15 \mathrm{E}-05$ \\
\hline 33 & Regulation of Rho protein signal transduction & & Intracellular & $5.54 \mathrm{E}-05$ \\
\hline 68 & Regulation of transcription, DNA-dependent & Transcription regulator activity, catalytic activity & Intracellular & $7.75 \mathrm{E}-05$ \\
\hline 29 & & Anion transporter activity, nucleic acid binding & & $2.82 \mathrm{E}-04$ \\
\hline 31 & Immune response & Obsolete molecular function & Extracellular region & $3.21 \mathrm{E}-04$ \\
\hline 7 & & & Intracellular, cell fraction & $1.01 \mathrm{E}-03$ \\
\hline 57 & & Transcription regulator activity, nucleotide binding & Extracellular region & $1.08 \mathrm{E}-03$ \\
\hline 6 & Regulation of biological process & ARF guanyl-nucleotide exchange factor activity & & $1.13 \mathrm{E}-03$ \\
\hline 66 & $\begin{array}{l}\text { Bone resorption, signal transduction, response to } \\
\text { stimulus }\end{array}$ & & & $1.47 \mathrm{E}-03$ \\
\hline 47 & & & $\begin{array}{l}\text { Peripheral to membrane of } \\
\text { membrane fraction }\end{array}$ & $2.34 \mathrm{E}-03$ \\
\hline 2 & $\begin{array}{l}\text { Response to stimulus, protein targeting, } \\
\text { regulation of biological process }\end{array}$ & Signal transducer activity & Cell & $2.84 \mathrm{E}-03$ \\
\hline 8 & Protein targeting & Transcription regulator activity & Intracellular & $3.26 \mathrm{E}-03$ \\
\hline 12 & & Catalytic activity, transporter activity & Extracellular region & $4.11 \mathrm{E}-03$ \\
\hline 15 & $\begin{array}{l}\text { Protein metabolism, regulation of biological } \\
\text { process }\end{array}$ & Catalytic activity, transporter activity & Membrane & $5.39 \mathrm{E}-03$ \\
\hline 17 & & GTPase activator activity, binding & Intracellular & $1.07 \mathrm{E}-02$ \\
\hline 16 & & $\begin{array}{l}\text { Protein binding, nucleotide binding, ATP-dependent helicase } \\
\text { activity, nucleic acid binding }\end{array}$ & & $1.08 \mathrm{E}-02$ \\
\hline 76 & & Nucleotide binding & $\begin{array}{l}\text { Cytoplasm, integral to } \\
\text { membrane }\end{array}$ & $1.09 \mathrm{E}-02$ \\
\hline 70 & & & $\begin{array}{l}\text { Extracellular region, membrane } \\
\text { fraction }\end{array}$ & $1.33 \mathrm{E}-02$ \\
\hline 53 & Regulation of transcription, DNA-dependent & ATP binding & & $1.71 \mathrm{E}-02$ \\
\hline 67 & & Protein phosphatase type $2 \mathrm{C}$ activity & & $1.84 \mathrm{E}-02$ \\
\hline 55 & Regulation of transcription, DNA-dependent & $\begin{array}{l}\text { Nucleotide binding, nucleic acid binding, ATP-dependent } \\
\text { helicase activity }\end{array}$ & & $1.89 \mathrm{E}-02$ \\
\hline
\end{tabular}


containing 11 genes as in the tested solution. The best (minimum) $p$-value obtained has been 0.008 , and the deepest level in the ontologies has been 2 , very generic terms. Therefore, we can conclude that the genes selected by TriGen have a closer relation in terms of GO annotations than expected if they were selected at random.

\section{Conclusions}

We have presented TriGen, a triclustering algorithm based on an evolutionary heuristic, genetic algorithms, which finds groups of pattern similarity for genes on a three dimensional space, thus taking into account the gene, conditions and time factor. The genetic operators used have been described in detail, along with the two fitness functions available to use with TriGen: $f_{M S R}$ based on a three-dimensional adaptation of the Mean Square Residue measure, a classic biclustering distance measure and $f_{L S L}$, a correlation measure based on the distance among the slopes of the least square lines from the three views of a tricluster.

The algorithm has been applied to three different datasets: a synthetic dataset, a dataset from a yeast cell cycle experiment and a dataset from the inflammation and host response to injury. The results show that the algorithm finds genes with high similarity in a subset of conditions and times. Moreover, the genes grouped together by the TriGen algorithm showed to be biologically related in terms of the functional annotations associated to them in the Gene Ontology project. We have also proved that genes selected by TriGen have a closer relation in terms of GO annotations than expected if they were selected at random. We have also seen that the two evaluation functions provide complementary information, since the overlapping of genes between triclusters found by $f_{M S R}$ and by $f_{L S L}$ is very low $(0.07 \%$ for the yeast cell cycle problem and $1.5 \%$ for the inflammation and host response to injury problem).

TriGen is an algorithm created to mine longitudinal experiments with microarray data, but it can be used in other biologically related fields, for instance combining expression data with gene regulation information by means of substituting the time dimension by ChIP-chip data representing transcription factorgene interactions what can provide us with regulatory network information. This proposal can also be applied to mine RNA-seq data repositories. Also, the seismic regionalization of the Iberian Peninsula is currently being addressed through TriGen, turning the 2D-dimensional problem proposed in [27] into a 3D one. In this case, the third component does not identify time stamps but features associated to every pair of geographical coordinates that represent the Iberian Peninsula.

\section{Acknowledgments}

The authors want to thank the financial support given by the Spanish Ministry of Science and Technology, projects TIN201128956-C00 and TIN2009-13950. This work has also been partially funded by Junta de Andalucía's project TIC-7528 and by JPI 2012 Banco Santander's grant.

\section{References}

[1] R.B. Araújo, G.H.T. Ferreira, G.H. Orair, W. Meira, R.A.C. Ferreira, D.O.G. Neto, M.J. Zaki, The ParTriCluster algorithm for gene expression analysis, Int. J. Parallel Progr. 36 (2) (2008) 226-249.

[2] M. Ashburner, C.A. Ball, J.A. Blake, D. Botstein, H. Butler, J.M. Cherry, A.P. Davis, K. Dolinski, S.S. Dwight, J.T. Eppig, M.A. Harris, D.P. Hill, L. Issel-Tarver, A. Kasarskis, S. Lewis, J.C. Matese, J.E. Richardson, M. Ringwald, G.M. Rubin, G. Sherlock, Gene ontology: tool for the unification of biology. The Gene ontology consortium, Nat. Genet. 25 (1) (2000) 9.
[3] H. Banka, S. Mitra, Evolutionary biclustering of gene expressions, Ubiquity 5 (2006) 2006

[4] Z. Bar-Joseph, Analyzing time series gene expression data, Bioinformatics 20 (16) (2004) 2493.

[5] M. Barenco, J. Stark, D. Brewer, D. Tomescu, R. Callard, M. Hubank, Correction of scaling mismatches in oligonucleotide microarray data, BMC Bioinforma. 7 (2006) 251.

[6] A. Ben-Dor, B. Chor, R. Karp, Z. Yakhini, Discovering local structure in gene expresión data: the order-preserving submatrix problem, in: Proceedings of the 6th International Conference on Computational Biology, 2002, pp. 49-57.

[7] P. Brown, D. Botstein, Exploring the new world of the genome with DNA microarrays, Nat. Genet. 21 (Suppl.) (1999) 33-37.

[8] S.E Calvano, W Xiao, D.R Richards, RM. Felciano, H. Baker, RJ Cho, R.O. Chen, B.H. Brownstein, J.P. Cobb, S.K. Tschoeke, C. Miller-Graziano, L. L. Moldawer, M.N. Mindrinos, R.W. Davis, R.G. Tompkins, S.F. Lowry, I.A. Large Scale Collab Res Program, A network-based analysis of systemic inflammation in humans, Nature 437 (2005) 1032-1037.

[9] Y. Cheng, G.M. Church, Biclustering of expression data, in: Internationa Conference on Intelligent Systems for Molecular Biology, 2000, pp. 93-103.

[10] P. D'haeseleer, S. Liang, R. Somogyi, Genetic network inference: from coexpression clustering to reverse engineering, Bioinformatics 16 (8) (2000) 707-726.

[11] R. Durbin, S. Eddy, A. Krogh, G. Mitchison, Biological Sequence Analysis: Probabilistic Models of Proteins and Nucleic Acids, Cambridge University Press, 1998.

[12] G. Getz, E. Levine, E. Domany, Coupled two-way clustering analysis of gene microarray data, Proc. Natl. Acad. Sci. 97 (22) (2000) 12079-12084.

[13] F. Gómez-Vela, F. Martínez-Álvarez, C.D. Barranco, N. Díaz-Díaz, D.S. Rodríguez-Baena, J.S. Aguilar-Ruiz, Pattern recognition in biological time series, in: Lecture Notes in Artificial Intelligence, vol. 7023, 2011, pp. 164-172.

[14] D. Gutiérrez-Avilés, C. Rubio-Escudero, J.C. Riquelme, Revisiting the yeast cell cycle problem with the improved trigen algorithm, in: IEEE World Congress on Nature and Biologically Inspired Computing, 2011, pp. 515-520.

[15] K. Hakamada, M. Okamoto, T. Hanai, Novel technique for preprocessing high dimensional time-course data from DNA microarray: mathematical modelbased clustering, Bioinformatics 22 (7) (2006) 843.

[16] J.A. Hartigan, Direct clustering of a data matrix, J. Am. Stat. Assoc. 67 (337) (1972) 123-129.

[17] Z. Hu, R. Bhatnagar, Algorithm for discovering low-variance 3-clusters from real-valued datasets, in: IEEE International Conference on Data Mining, 2010 pp. 236-245.

[18] H. Jiang, S. Zhou, J. Guan, Y. Zheng, gTRICLUSTERL: a more general and effective 3D clustering algorithm for gene-sample-time microarray data, in: Lecture Notes in Computer Science, vol. 3916, 2006, pp. 48-59.

[19] Z. Li, X. Hu, Y. Chen, Multi-objective evolutionary algorithm for mining 3D clusters in gene-sample-time microarray data, in: IEEE International Conference on Granular Computing, 2008, pp. 442-447.

[20] Y.C. Liu, C.H. Lee, W.C. Chen, J.W. Shin, H.H. Hsu, V.S. Tseng, A novel method for mining temporally dependent association rules in three-dimensional microarray datasets, in: IEEE International Computer Symposium, 2010, pp. 759-764.

[21] S.C. Madeira, A.L. Oliveira, Biclustering algorithms for biological data analysis: a survey, IEEE/ACM Trans. Comput. Biol. Bioinform. 1 (1) (2004) 24-45.

[22] P. Mahanta, H.A. Ahmed, D.K. Bhattacharyya, J.K. Kalita, Triclustering in gene expression data analysis: a selected survey, IEEE International Conference on Emerging Trends and Applications in Computer Science, 2011, pp. 1-6.

[23] M. Martínez-Ballesteros, F. Martínez-Álvarez, A. Troncoso, J.C. Riquelme, An evolutionary algorithm to discover quantitative association rules in multidimensional time series, Soft Comput. 15 (2011) 2065-2084.

[24] P. Mendes, GEPASI: a software package for modelling the dynamics, steady states and control of biochemical and other systems, Comput. Appl. Biosci. 9 (2003) 563-571.

[25] S. Mitra, H. Banka, Multi-objective evolutionary biclustering of gene expression data, Pattern Recognit. 39 (12) (2006) 2464-2477.

[26] R.P. Pargas, M.J. Harrold, R.R. Peck, Test-data generation using genetic algorithms, Softw. Test. Verif. Reliab. 9 (4) (1999) 263-282.

[27] J. Reyes, V. Cárdenas, A Chilean seismic regionalization through a Kohonen neural network, Neural Comput. Appl. 19 (2010) 1081-1087.

[28] R. Romero-Záliz, C. Del Val, J.P. Cobb, I. Zwir, Onto-cc: a web server for identifying gene ontology conceptual clusters, Nucl. Acids Res. 36 (Suppl. 2) (2008) W352-W357.

[29] R. Romero-Záliz, C. Rubio-Escudero, J.P. Cobb, F. Herrera, O. Cordón, I. Zwir A multiobjective evolutionary conceptual clustering methodology for gene annotation within structural databases: a case of study on the Gene Ontology database, IEEE Trans. Evol. Comput. 12 (6) (2008) 679-701.

[30] C. Rubio-Escudero, Fusion of knowledge towards the identification of genetic profiles, AI Commun. 25 (1) (2012) 65-67.

[31] C. Rubio-Escudero, F. Martínez-Álvarez, R. Romero-Záliz, I. Zwir, Classification of gene expression profiles: comparison of K-means and expectation maximization algorithms, in: Proceedings of the IEEE International Conference on Hybrid Intelligent Systems, 2008, pp. 831-836.

[32] K. Sim, Z. Aung, Discovering correlated subspace clusters in 3D continuous-valued data, in: IEEE International Conference on Data Mining, 2010, pp. 471-480.

[33] P.T. Spellman, G. Sherlock, M.O. Zhang, V.R. Iyer, K. Anders, M.B. Eisen, P.O. Brown, D. Botstein, B. Futcher, Comprehensive identification of cell cycle-regulated genes of the yeast Saccharomyces cerevisiae by microarray hybridization, Mol. Biol. Cell 9 (3) (1998) 3273-3297. 
[34] M.P. Tan, E.N. Smith, J.R. Broach, C.A. Floudas, Microarray data mining: a novel optimization-based approach to uncover biologically coherent structures, BMC Bioinforma. 9 (2008) 268.

[35] A. Tanay, R. Sharan, R. Shamir, Discovering statistically significant biclusters in gene expression data, Bioinformatics 18 (Suppl. 1) (2002) S136-S144.

[36] A.B. Tchagang, S. Phan, F. Famili, H. Shearer, P. Fobert, Y. Huang, J. Zou, D. Huang, A. Cutler, Z. Liu, Mining biological information from 3D short timeseries gene expression data: the OPTricluster algorithm, BMC Bioinforma. 13 (1) (2012) 54.

[37] R. Tibshirani, T. Hastie, M. Eisen, D. Ross, D. Botstein, P. Brown, et al., Clustering Methods for the Analysis of DNA Microarray Data, Technical Report, Department of Statistics, Stanford University, 1999.

[38] G. Wang, L. Yin, Y. Zhao, K. Mao, Efficiently mining time-delayed gene expression pattern, IEEE Trans. Syst. Man Cybern. Part B: Cybern. 40 (2) (2010) 400-411.

[39] X. Xu, Y. Lu, K.L. Tan, A. Tung, Finding time-lagged 3D clusters, in: IEEE International Conference on Data Engineering, 2009, pp. 445-456.

[40] Y. Yin, Y. Zhao, B. Zhang, G. Wang, Mining time-shifting co-regulation patterns from gene expression data, in: Lecture Notes in Computer Science, vol. 4505, 2007, pp. 62-73.

[41] L. Zhao, M.J. Zaki, TRICLUSTER: an effective algorithm for mining coherent clusters in 3D microarray data, in: ACM SIGMOD International Conference on Management of Data, 2005, pp. 694-705. 